\title{
Phantom Study of a New Laser-Etched Needle for Improving Visibility During Ultrasonography-Guided Lumbar Medial Branch Access With Novices
}

\author{
Jung Wook Park, $\mathrm{MD}^{1}$, Min Woo Cheon, $\mathrm{PhD}^{2}$, Min Hong Lee, $\mathrm{MD}^{3}$
}

${ }^{1}$ Topteam Rehabilitation Clinic, Gwangju; ${ }^{2}$ Department of Hospital Biomedical Engineering, Dongshin University, Gwangju; ${ }^{3}$ Department of Rehabilitation Medicine, Chosun University Hospital, Gwangju, Korea

\begin{abstract}
Objective To compare the visibility and procedural parameters between a standard spinal needle and a new laser-etched needle (LEN) in real-time ultrasonography guided lumbar medial branch access in a phantom of the lumbosacral spine

Methods We conducted a prospective single-blinded observational study at a rehabilitation medicine center. A new model of LEN was manufactured with a standard 22-gauge spinal needle and a laser etching machine. Thirtytwo inexperienced polyclinic medical students performed ultrasonography-guided lumbar medial branch access using both a standard spinal needle and a LEN with scanning protocol. The outcomes included needle visibility score, needle elapsed time, first-pass success rate, and number of needle sticks.

Results The LEN received significantly better visibility scores and shorter needle elapsed time compared to the standard spinal needle. First-pass success rate and the number of needle sticks were not significantly different between needles.

Conclusion A new LEN is expected to offer better visibility and enable inexperienced users to perform an ultrasonography-guided lumbar medial branch block more quickly. However, further study of variables may be necessary for clinical application.
\end{abstract}

Keywords Zygapophyseal joint, Ultrasonography, Needles, Radiologic phantoms, Injections

\footnotetext{
Received August 18, 2015; Accepted September 30, 2015

Corresponding author: Min Hong Lee

Department of Rehabilitation Medicine, Chosun University Hospital, 365 Pilmun-daero, Dong-gu, Gwangju 61453, Korea. Tel: +82-62-974-9191, Fax: +82-62-223-0245, E-mail: hayaaaa@hanmail.net

ORCID: Jung Wook Park (http://orcid.org/0000-0002-2507-6111); Min Woo Cheon (http://orcid.org/0000-0002-0979-4316); Min Hong Lee (http:// orcid.org/0000-0002-4457-5434).
}

() This is an open-access article distributed under the terms of the Creative Commons Attribution Non-Commercial License (http://creativecommons.org/ licenses/by-nc/4.0) which permits unrestricted noncommercial use, distribution, and reproduction in any medium, provided the original work is properly cited. Copyright $\odot 2016$ by Korean Academy of Rehabilitation Medicine 


\section{INTRODUCTION}

Lumbar facet joint-mediated pain is considered one of the major causes of chronic low back pain. However, it is not enough to confirm the diagnosis with physical examination or radiographic imaging [1]. Currently, selective anesthesia of the medial branch of the dorsal primary ramus of the spinal nerve innervating the facet joint is the accepted standard to diagnose facet joint-mediated pain [2]. Recent multiple studies have demonstrated that the success rate of ultrasonography (US)-guided lumbar medial branch block is not lower than fluoroscopy-guided block $[1,3,4]$. This development has been shown to be safe and yields better efficacy due to elimination of radiation exposure, ensuring accurate deposition and spread of local anesthetic around the target nerve [5]. Despite the numerous advantages of the use of US guidance, disruption of the image of the needle tip driven toward the target is a general problem, especially for unskilled users; the success and safety of US-guided procedures are highly dependent on needle visualization $[6,7]$. Lack of visualization of the needle during advancement in lumbar spine injections may contribute to inadvertent damage of other tissues, puncture of paraspinal vessels, and spinal anesthesia even with experienced operators $[8,9]$. Therefore, a number of methods have been used to enhance echogenicity to increase needle visualization during US using different technologies and designs [10-13]. According to Edgcombe and Hocking [14], it has been shown that such echo-enhanced needles improve identification of the needle tip by both experienced and inexperienced operators. Although commercially available echo-enhanced needles have distinct advantages, they are more expensive than corresponding non-echogenic needles [15]. Therefore, we developed a new echo-enhanced needle with a modified surface using a standard spinal needle with a laser etching machine for lumbar spine injection. The purpose of this study was to compare the visibility and procedural parameters between a standard spinal needle and a new laser-etched needle (LEN) in real-time US-guided lumbar medial branch access in a lumbosacral spine phantom by novice operators.

\section{MATERIALS AND METHODS}

\section{Subjects}

A prospective single-blinded observational study was conducted at a rehabilitation medicine center. Thirty-two polyclinic medical students were invited to participate in this study; they had no prior experience of US-guided procedures.

\section{Study equipment}

The LEN was manufactured using a standard 22-gauge spinal needle (Quincke-Type Point 3.5 inches; BD Medical, Franklin Lakes, NJ, USA) by laser etching machine (fibered diode laser, FDL; Datalogic, Bologna, Italy). Two ultrasound reflectors were laser-etched precisely next to the surface of the needle tip with $2 \mathrm{~mm}$ intervals and 5 ultrasound reflectors were positioned equally spaced ( 5 $\mathrm{mm}$ apart) on the same side of the distal needle shaft (Fig. 1).

The ultrasound phantom of the lumbosacral spine was made by following the method proposed by Bellingham and Peng [16], using an adult-size spine model consisting of the lower 3 lumbar vertebra and sacrum with only the bony elements. Sugar-free Metamucil (P\&G, Cincinnati, $\mathrm{OH}, \mathrm{USA}$ ) was added to the surface layer of gelatin in the phantom (Fig. 2). This gelatin-Metamucil mixture simulated the sonographic imaging of subcutaneous soft tissue and made the surface layer of the phantom opaque. Thus, the needle and targets were only visible with US, not with the naked eye [17]. For US, the Accuvix V10 (Medison, Seoul, Korea) was used with a 2-6 MHz curved array transducer.

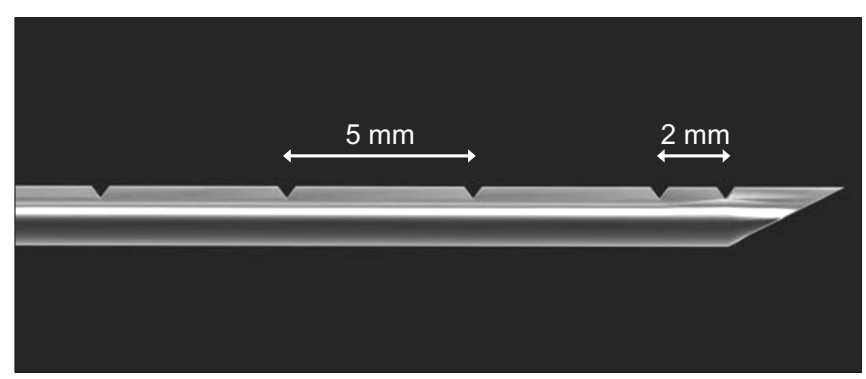

Fig. 1. Computer graphic image of laser-etched needle shows two ultrasound reflectors adjacent to the surface of the needle tip at $2 \mathrm{~mm}$ intervals and same-shape ultrasound reflectors located at $5 \mathrm{~mm}$ intervals on the same side of the distal needle shaft. 


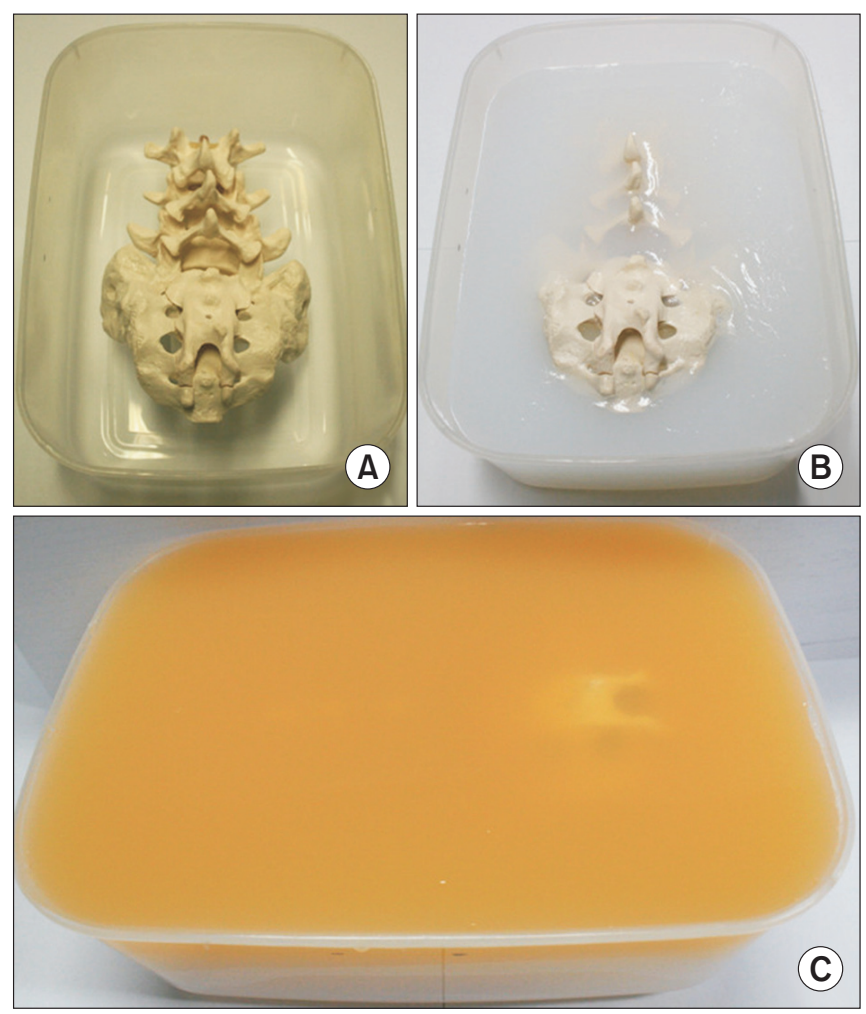

Fig. 2. Gelatin-Metamucil mixture of the lumbosacral spine phantom. (A) An adult-size lumbosacral spine model. (B) Spinal phantom after the lower layer of gelatin mold has set. (C) Yellowish surface layer of the spinal phantom after the Metamucil-mixed gelatin mold has been added.

\section{Method}

Prior to attempting the procedure, all participants were presented with a 60-minute lecture on the proposed procedure including spinal anatomy and scanning protocols. The instruction included holding the probe on the phantom, visualization of the target point, and needle visualization using the in-plane technique. Subsequently, participants received training in real-time US-guided needle handling with pork meat in such a manner that the needle shaft and the tip were visible in-plane to the ultrasound beam for 20 minutes [15]. After the training session, a random-number generator was used to assign which needle the participant started with ( 1 , standard spinal needle; 2, LEN). All participants were asked to perform real-time US-guided third lumbar medial branch access using both a standard 22-gauge spinal needle and a LEN using the in-plane approach in a lumbosacral spine phantom.
In the protocol, the target point for third lumbar medial branch access was defined as lying on the upper edge of the fourth lumbar transverse process and in the groove at the base of the superior articular process [1]. On the lumbosacral spine phantom, posterior paravertebral parasagittal sonograms with a long-axis view were obtained to identify the fourth lumbar vertebra by counting the transverse processes from the sacrum upward. Then, the transducer was positioned in a transverse plane to delineate the transverse processes and the superior articular processes of the adjacent facet joint [18]. After orienting the ultrasound probe toward the target point, the needle was inserted $6 \mathrm{~cm}$ lateral to the midline at the lateral end of the transducer and positioned in the ultrasound plane at a depth of $5 \mathrm{~cm}$ and an angle of approximately $45^{\circ}-50^{\circ}$ to the surface (Fig. 3). Subsequently, the needle was advanced in a lateral to medial direction until the needle tip reached the target and bony contact occurred. The inserted needle was left in this position and a well-experienced investigator confirmed the successful placement of the needle tip with both in-plane and out-of-plane approaches. Then participants repeated the same process with the other needle.

All participants were blinded to the needle type while performing this procedure and were prohibited from observing other participants' procedures. They were directly observed by an investigator during the procedure and the time to achieve successful needle-target contact was recorded. All needle passes were saved on the hard disk of the ultrasound device.

\section{Outcome measures}

After performing the procedure, all participants were asked to subjectively score needle visibility on a scale from 1 to 5 (1, very poor; 2 , poor; 3 , fair; 4 , good; 5 , very good) during their own performance $[7,19]$. Needle elapsed time was measured from the moment of needle stick into the phantom tissue and terminated when proper bony contact of the needle tip was visualized by ultrasound. When needle-target contact was incorrect, operators were allowed to attempt the procedure again and outcome measurements were recorded upon success. Number of needle sticks were recorded to evaluate first-pass success rates. 

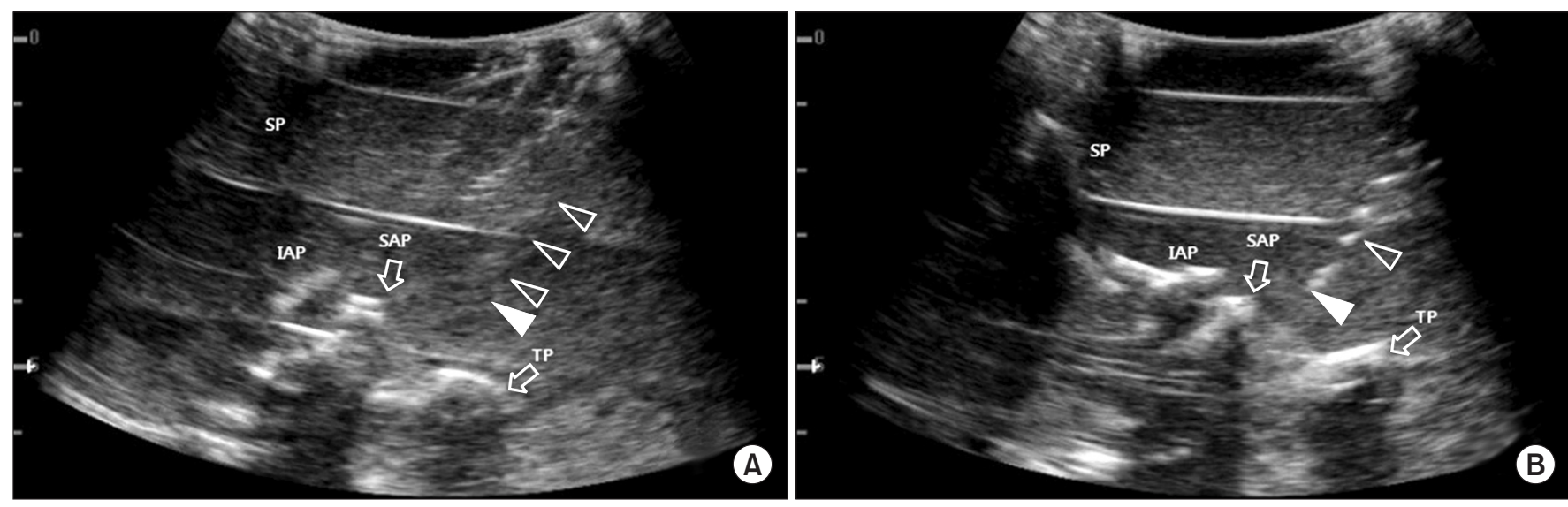

Fig. 3. Ultrasound images of right third lumbar medial branch access with study needles in lumbosacral spine phantom. (A) Using the standard spinal needle. (B) The image of laser-etched needle shows bright dots from the ultrasound reflectors. Open arrowheads, needle shaft; closed arrowheads, needle tip; SP, spinous process; TP, transverse process; IAP, inferior articular process; SAP, superior articular process.

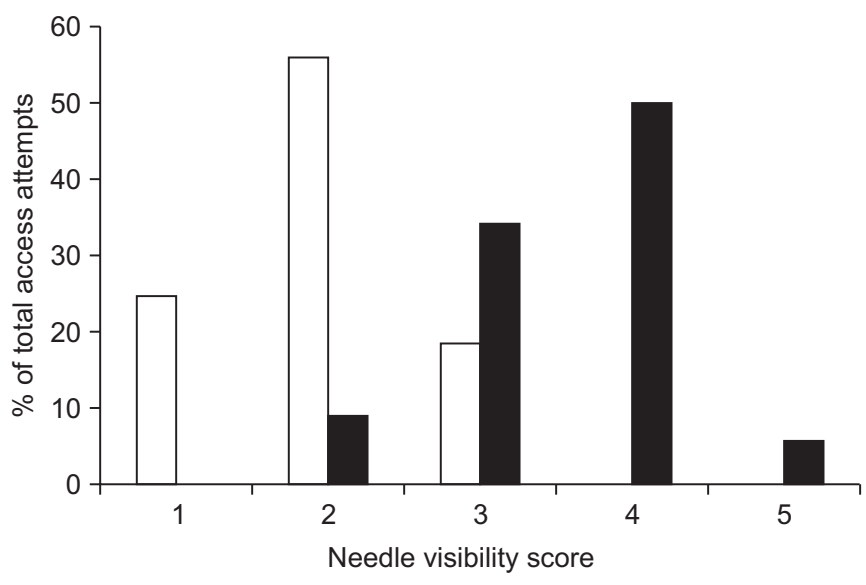

Fig. 4. Estimates of needle visibility for standard spinal needle (gray bars) and laser-etched needle (black bars).

\section{Statistical analysis}

Statistical analysis was performed with IBM SPSS Statistics ver. 20 (IBM, Armonk, NY, USA). The Mann-Whitney $\mathrm{U}$ test was used for comparison of needle visibility, needle elapsed time and number of needle sticks between the two groups. Fisher exact test was used to compare needle first-pass success rates. The p-values less than 0.05 were considered statistically significant. Analyzed data are presented as mean values with standard deviation and/or range of $95 \%$ confidence intervals (CI).

\section{RESULTS}

A total of 64 US-guided third lumbar medial branch ac- cess procedures were performed on lumbosacral phantoms by 32 participants. Data of needle visibility is shown in Fig. 4. The LEN received significantly better visibility scores relative to the standard needle -3.53 (95\% CI, 3.26-3.79) vs. 1.94 (95\% CI, 1.71-2.17); $\mathrm{p}<0.001$. Needle elapsed time was shorter with the LEN also-13.18 seconds (95\% CI, 12.00-14.47) vs. 17.01 seconds (95\% CI, 15.65-18.47); $\mathrm{p}<0.001$. In 5 cases with the LEN, 2 needle stick attempts were made relative to the 9 cases of the standard spinal needle, where 3 needle stick attempts were made. First-pass success rate was not significantly different between needles-84.4\% (95\% CI, 70.6\%-96.4\%) vs. $65.6 \%$ (95\% CI, $48.5 \%-81.8 \%$ ); $\mathrm{p}=0.148$, and there were also no significant differences in number of needle sticks between groups-1.16 (95\% CI, 1.04-1.29) vs. 1.41 (95\% CI, 1.21-1.63), $\mathrm{p}=0.072$ (Table 1).

\section{DISCUSSION}

Recently, US-guided lumbar medial branch blocks have been demonstrated successfully except in obese patients $[3,18,20]$. The success and validity of lumbar medial branch blocks depends on accurate technique and needle visualization because of the small volume of local anesthetic administered to ensure specificity [4]. Inaccurate positioning of the needle may result in falsepositive blocks because of inadvertent spread of local anesthetic into the inter-vertebral foramen, the epidural space, or even the subarachnoid space, resulting in pos- 
Table 1. Comparison of outcome measures between LEN and SSN

\begin{tabular}{|c|c|c|c|c|c|}
\hline & \multicolumn{2}{|c|}{ LEN (n=32) } & \multicolumn{2}{|c|}{ SSN $(n=32)$} & \multirow{2}{*}{ p-value } \\
\hline & Mean \pm SD & $95 \% \mathrm{CI}$ & Mean \pm SD & $95 \% \mathrm{CI}$ & \\
\hline Visibility score & $3.53 \pm 0.76$ & $3.26-3.79$ & $1.94 \pm 0.67$ & $1.71-2.17$ & $<0.001^{\text {a) }}$ \\
\hline Needle elapsed time (s) & $13.18 \pm 3.60$ & $12.00-14.47$ & $17.01 \pm 4.09$ & $15.65-18.47$ & $<0.001^{\text {a) }}$ \\
\hline First-pass success rate (\%) & $84.4(27 / 32)$ & $70.6-96.4$ & $65.6(21 / 32)$ & $48.5-81.8$ & $0.148^{\mathrm{b})}$ \\
\hline Number of needle sticks & $1.16 \pm 0.37$ & $1.04-1.29$ & $1.41 \pm 0.62$ & $1.21-1.63$ & $0.072^{\text {a) }}$ \\
\hline
\end{tabular}

LEN, laser-etched needle; SSN, standard spinal needle; CI, confidence interval.

${ }^{a}$ Mann-Whitney U test, ${ }^{\text {b) }}$ Fisher exact test.

sible complications such as spinal anesthesia [8]. Thus, to enhance needle visibility on US-guided procedures, needle modifications such as polymer coating and tip dimpling have been made $[10,11]$. Thin polymeric coating film is smooth to the touch but rough microscopically. Small craters in the surface of the polymeric-coated needle trap micro-bubbles of air and increase reflectivity of ultrasound waves. Roughening the needle surface (dimpling) is a primitive effort in an attempt to alter the smooth surface of metal needles. However, polymericcoated needles have limited usage times due to a dense cloud of micro-bubbles that are absorbed by the surrounding fluid and tissue, and dimpled tip needles are limited by low shaft visibility and remain difficult to see in many locations [11]. Taking a step forward, specific needle surface modifications allowing ultrasound reflection irrespective of angle of orientation have been recently developed in an attempt to enhance needle visualization in US for regional blocks [7,14,21]. Sviggum et al. [22] reported that the SonoPlex Stim needle (Pajunk Medical Systems, Tucker, GA, USA) was rated highest for brightness and clarity in a gel phantom compared to several echo-enhanced needles. The SonoPlex Stim uses contains a texturized needle surface with multiple flat, angled laser-based markings called 'Cornerstone' reflectors. These indented regions increase the surface area of the needle across which the waves are reflected. These indentations between the smooth surfaces cause intense waves reflecting at different angles to bounce back to the probe by changing the reflected angles of the waves coming from the transducer [7,19,22]. Gofeld et al. [23] also noted that SonoPlex Stim appeared to have better needle tip visibility in their human cadaveric study followed by EchoStim (Havel Inc., Cincinnati, OH, USA) among representative commercially available echogenic needles in the United States. EchoStim has three similar angled indentations called 'corner cube reflectors (CCR)' on the distal shaft. The first reflector is located $2 \mathrm{~mm}$ from the distal end, with two more at $1 \mathrm{~mm}$ intervals; the needle tip appears as three hyper-echoic dots under US. However, CCRs are limited to the distal $4 \mathrm{~mm}$ of the needle in an effort to better identify its tip [21]. All these specific characteristics are designed to reflect a greater percentage of the ultrasound beam striking the needle back to the ultrasound probe at steeper insertion angles in diverse USguided regional anesthesia. Previous studies investigating such disposable echogenic needles have found that they provided outstanding visibility compared with standard needles $[14,19,22,23]$. Although all these needles have the potential to enhance needle echogenicity, economic factors may impede the routine use of echo-enhanced needles. Therefore, we manufactured a new echo-enhanced needle at low cost using a standard spinal needle, with specific simplified wedge shaped reflectors that were designed to increase reflected ultrasound waves back to the probe (Fig. 5).

In this study, we described the comparison between a new echo-enhanced needle and a standard spinal needle using a subjective measurement of visibility and needle elapsed time on dynamic clinical imaging with a group of novice operators using a lumbosacral spine phantom. Many studies use a subjective scoring system for assessment of needle visibility $[10,24,25]$. Currently, several studies have suggested objective measures of needle visibility in static images $[11,14,26,27]$. This method enables all observing clinicians to view and rate identical images. On the other hand, it is not able to provide additional information about needle position through other factors such as tissue movement in actual clinical situations. Besides, standardized methods for assessment of needle visibility do not exist [28]. The present study used the method defined by Kilicaslan et al. [7] to evaluate subjec- 

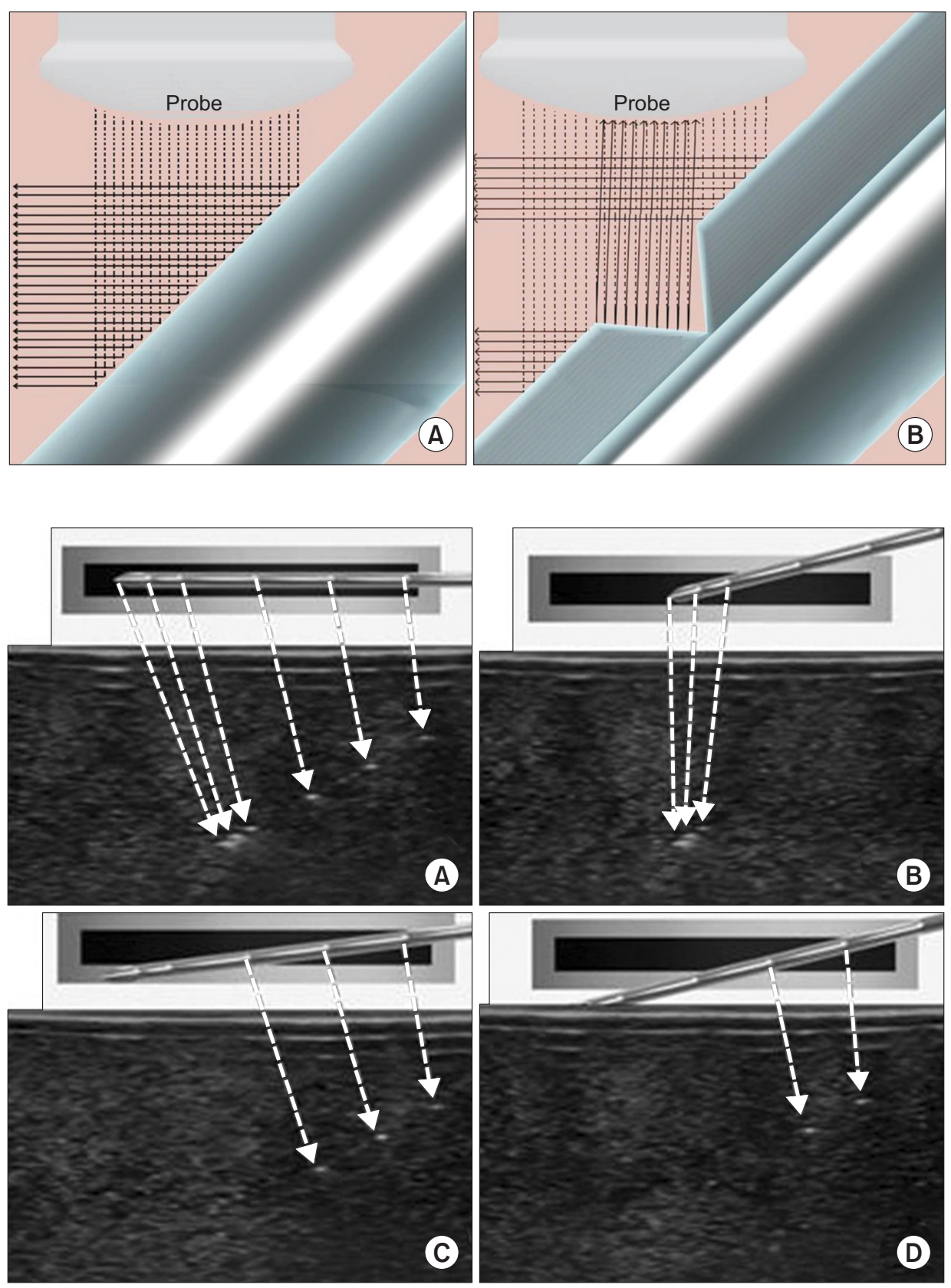

Fig. 5. Schematic diagram of ultrasound performance with the inplane approach. (A) Ultrasound beam is reflected off of standard spinal needle surface away from probe. (B) Due to wedge shaped reflectors, part of the ultrasound beam is reflected back to the probe.

Fig. 6. Ultrasound images of laseretched needle by location and direction of ultrasound beam (black bar) in soft tissue phantom. Dotted arrows, reflectors of laseretched needle. tive scoring for assessment of needle visibility in dynamic images.

Our study findings demonstrate improved visualization of the needle during lumbar medial branch access with LENs compared to standard spinal needles. Even though speed is not a priority, reduction of needle elapsed time may be the substitutionary measurement that reflects better applicability of the procedure. Higher first-pass success rates and a smaller number of needle insertions may signify the appropriateness of the procedure and potential decrease of iatrogenic injury rate, although these difference were not significant in our study.

Failure to visualize the needle was the most common error observed in novices learning to perform US-guided injection. Persistent failure to visualize the needle was documented even after performing more than one hundred US-guided peripheral nerve blocks, suggesting that experienced practitioners may face difficulties as well [9]. The main reason is the difficulty of maintaining parallelism of the transducer relative to needle trajectory and keeping the needle tip in view as the needle is advanced toward the target [29]. The afore-mentioned wedge 
shaped reflectors improve visualization of the needle tip and shaft; the needle tip and two shaped reflectors ( $2 \mathrm{~mm}$ apart) adjacent to the needle tip are visualized as dense bright dots on the ultrasound image, enabling identification of tip position even if the needle shaft is not completely seen. Additionally, 5 reflectors on the needle shaft ( $5 \mathrm{~mm}$ apart) are intended to enable the users to more simply optimize the shaft visibility when the angle of the ultrasound beam is unintentionally oblique relative to the inserted needle (Fig. 6). These considerations are able to offer a three-dimensional spatial relationship of the inserted needle to users and will likely lead to decreased proportion of iatrogenic injuries to neural or other surrounding structures.

This study has several limitations including difficulty in blinding the needle type, because the 2 needles appear different sonographically. If a good in-plane image was obtained, then the needle in use may have been evident to the participants. Secondly, we did not separate needle tip and shaft visibility. This methodology has been used formerly by Guo et al. [19] and allows comparison of the overall visibility of the needles. Thirdly, we did not compare different types of other echo-enhanced needles. It seems to be necessary to compare the other commercially available echo-enhanced needles with LENs to acquire objective validity. The fact that this study was performed by inexperienced polyclinic medical students also poses a limitation. It is logical to expect that novices would benefit more than expert sonographers from using echo-enhanced needles. But in previous studies, there were no significant differences between novice and expert groups in subjective needle-visibility with variant echogenic needles $[13,14]$. Nevertheless, further study with well-experienced clinicians might be necessary for clinical application. Lastly, we conducted the experiments at one angle of needle insertion in a phantom. Several studies have shown that needle visibility becomes poor as the angle of needle insertion increases in relation to the ultrasound probe due to reflective signal losses $[26,27]$. Furthermore, for variable spinal procedures and another regional anesthesia with US, studies of shallow and steeper angle approaches with the LEN are required. However, the choice of US-guided lumbar medial branch access in a phantom was challenging because of a unique combination of accompanying difficulties, such as deep tissue planes, fixed anatomical targets, steep procedural angles, and bone artifacts. This particular setting of deep access may be required for common paravertebral procedures and the methodology made an attempt to replicate clinical scenarios [23]. Despite phantoms not having the same echogenic characteristics as real tissues and structures, the use of phantoms allowed us to standardize the level of difficulty each participant encountered while performing US-guided lumbar medial branch access procedures. Nonetheless, further clinical studies conducted with cadavers that utilize a wide range of parameters on safety and efficacy will be helpful to determine the effects of needle visibility in novice users.

In conclusion, LENs provide significantly superior visibility compared to standard spinal needles during USguided lumbar medial branch access in a lumbar phantom. Additionally, inexperienced users who used the LEN were able to perform the procedure more rapidly. Moreover, the LEN is expected to define the three-dimensional spatial orientation of the inserted needle to the operator during US.

\section{CONFLICT OF INTEREST}

No potential conflict of interest relevant to this article was reported.

\section{REFERENCES}

1. Greher M, Scharbert G, Kamolz LP, Beck H, Gustorff B, Kirchmair L, et al. Ultrasound-guided lumbar facet nerve block: a sonoanatomic study of a new methodologic approach. Anesthesiology 2004;100:1242-8.

2. Saal JS. General principles of diagnostic testing as related to painful lumbar spine disorders: a critical appraisal of current diagnostic techniques. Spine (Phila Pa 1976) 2002;27:2538-45.

3. Ha DH, Shim DM, Kim TK, Kim YM, Choi SS. Comparison of ultrasonography- and fluoroscopy-guided facet joint block in the lumbar spine. Asian Spine J 2010;4:15-22.

4. Kaplan M, Dreyfuss P, Halbrook B, Bogduk N. The ability of lumbar medial branch blocks to anesthetize the zygapophysial joint: a physiologic challenge. Spine (Phila Pa 1976) 1998;23:1847-52.

5. Chin KJ, Perlas A, Chan VW, Brull R. Needle visualization in ultrasound-guided regional anesthesia: challenges and solutions. Reg Anesth Pain Med 2008;33:532-44. 
6. Crum T, Adhikari S, Lander L, Blaivas M. Do echoenhanced needles make a difference in sonographically guided vascular access? J Ultrasound Med 2014;33:623-8.

7. Kilicaslan A, Topal A, Tavlan A, Erol A, Otelcioglu S. Differences in tip visibility and nerve block parameters between two echogenic needles during a simulation study with inexperienced anesthesia trainees. J Anesth 2014;28:460-2.

8. Marks R, Semple AJ. Spinal anaesthesia after facet joint injection. Anaesthesia 1988;43:65-6.

9. Sites BD, Spence BC, Gallagher JD, Wiley CW, Bertrand ML, Blike GT. Characterizing novice behavior associated with learning ultrasound-guided peripheral regional anesthesia. Reg Anesth Pain Med 2007;32:107-15.

10. Gottlieb RH, Robinette WB, Rubens DJ, Hartley DF, Fultz PJ, Violante MR. Coating agent permits improved visualization of biopsy needles during sonography. AJR Am J Roentgenol 1998;171:1301-2.

11. Culp WC, McCowan TC, Goertzen TC, Habbe TG, Hummel MM, LeVeen RF, et al. Relative ultrasonographic echogenicity of standard, dimpled, and polymeric-coated needles. J Vasc Interv Radiol 2000;11:351-8.

12. Barr RG. Improved needle visualization with electronic beam steering: proof of concept. Ultrasound Q 2012;28:59-64.

13. Hocking G, Mitchell CH. Optimizing the safety and practice of ultrasound-guided regional anesthesia: the role of echogenic technology. Curr Opin Anaesthesiol 2012;25:603-9.

14. Edgcombe H, Hocking G. Sonographic identification of needle tip by specialists and novices: a blinded comparison of 5 regional block needles in fresh human cadavers. Reg Anesth Pain Med 2010;35:207-11.

15. Uppal V, Sondekoppam RV, Ganapathy S. Effect of beam steering on the visibility of echogenic and nonechogenic needles: a laboratory study. Can J Anaesth 2014;61:909-15.

16. Bellingham GA, Peng PW. A low-cost ultrasound phantom of the lumbosacral spine. Reg Anesth Pain Med 2010;35:290-3.

17. Kendall JL, Faragher JP. Ultrasound-guided central venous access: a homemade phantom for simulation. CJEM 2007;9:371-3.

18. Greher M, Kirchmair L, Enna B, Kovacs P, Gustorff
B, Kapral S, et al. Ultrasound-guided lumbar facet nerve block: accuracy of a new technique confirmed by computed tomography. Anesthesiology 2004;101:1195-200.

19. Guo S, Schwab A, McLeod G, Corner G, Cochran $S$, Eisma R, et al. Echogenic regional anaesthesia needles: a comparison study in Thiel cadavers. Ultrasound Med Biol 2012;38:702-7.

20. Rauch S, Kasuya Y, Turan A, Neamtu A, Vinayakan A, Sessler DI. Ultrasound-guided lumbar medial branch block in obese patients: a fluoroscopically confirmed clinical feasibility study. Reg Anesth Pain Med 2009;34:340-2.

21. Miura M, Takeyama K, Suzuki T. Visibility of ultrasound-guided echogenic needle and its potential in clinical delivery of regional anesthesia. Tokai J Exp Clin Med 2014;39:80-6.

22. Sviggum HP, Ahn K, Dilger JA, Smith HM. Needle echogenicity in sonographically guided regional anesthesia: blinded comparison of 4 enhanced needles and validation of visual criteria for evaluation. J Ultrasound Med 2013;32:143-8.

23. Gofeld M, Krashin DL, Ahn S. Needle echogenicity in ultrasound-guided lumbar spine injections: a cadaveric study. Pain Physician 2013;16:E725-30.

24. Deam RK, Kluger R, Barrington MJ, McCutcheon CA. Investigation of a new echogenic needle for use with ultrasound peripheral nerve blocks. Anaesth Intensive Care 2007;35:582-6.

25. Maecken T, Zenz M, Grau T. Ultrasound characteristics of needles for regional anesthesia. Reg Anesth Pain Med 2007;32:440-7.

26. Schafhalter-Zoppoth I, McCulloch CE, Gray AT. Ultrasound visibility of needles used for regional nerve block: an in vitro study. Reg Anesth Pain Med 2004;29:480-8.

27. Nichols K, Wright LB, Spencer T, Culp WC. Changes in ultrasonographic echogenicity and visibility of needles with changes in angles of insonation. J Vasc Interv Radiol 2003;14:1553-7.

28. Hebard S, Hocking G. Echogenic technology can improve needle visibility during ultrasound-guided regional anesthesia. Reg Anesth Pain Med 2011;36:1859.

29. Chapman GA, Johnson D, Bodenham AR. Visualisation of needle position using ultrasonography. Anaesthesia 2006;61:148-58. 